5. ATOMIC AND MOLECULAR DATA FOR ASTROCHEMISTRY

Scientific Organizing Committee

P.L. Smith (Chairman)

C. Arpigny, D. Flower

Supporting Commissions:

$$
14,15,29,34,40,44
$$

https://doi.org/10.1017/S1539299600007942 Published online by Cambridge University Press 


\title{
ATOMIC AND MOLECULAR DATA FOR DIFFUSE CLOUD CHEMISTRY
}

\author{
Ewine F. van Dishoeck \\ Div. of Geological and Planetary Sciences \\ California Institute of Technology 170-25 \\ Pasadena, CA 91125.
}

\begin{abstract}
A brief overview of the procedure for modeling diffuse interstellar clouds is given, and specific needs for atomic and molecular data are pointed out.
\end{abstract}

\section{INTRODUCTION}

Diffuse cloud chemistry is the chemistry of the simplest interstellar molecules. This results from the fact that diffuse clouds are very tenuous concentrations of the interstellar gas with total visual extinctions $A_{V}<2$ mag. The interstellar ultraviolet radiation can easily penetrate these diffuse clouds and prevent the build -up of large molecules through rapid photodestruction processes. Most elements therefore exist primarily in atomic form in diffuse clouds, and any atom with an ionization potential less than $13.6 \mathrm{eV}$ is mostly ionized. Thus carbon is present primarily as $\mathrm{C}^{+}$, oxygen as $\mathrm{O}$ and nitrogen as $\mathrm{N}$, with the most abundant molecule, $\mathrm{CO}$, containing at most $1 \%$ of the total carbon. The only exception is formed by hydrogen, where $\mathrm{H}_{2}$ may be comparable in abundance to $\mathrm{H}$. Because of their simplicity, diffuse clouds are thought to be the best environment in which to test the basic concepts of interstellar chemistry.

The chemistry in diffuse clouds has recently been reviewed in great detail by van Dishoeck and Black (1988a), van Dishoeck (1988a), Dalgarno (1988), Black (1987), van Dishoeck and Black (1986) and Crutcher and Watson (1985). Because of space limitations, only a brief summary is given here with limited references to the original papers. Emphasis is placed on specific needs for atomic and molecular data. The reader is referred to the above papers for more details.

\section{OBSERVATIONS}

Diffuse clouds are studied mostly by the absorption lines of the atoms and molecules superposed on the spectra of bright background stars. The classic example is the line of sight toward $\varsigma$ Oph. Although many atomic species have been identified, only few molecules have so far been found in diffuse clouds: $\mathrm{H}_{2}, \mathrm{HD}, \mathrm{CH}, \mathrm{CH}^{+}, \mathrm{C}_{2}, \mathrm{CO}, \mathrm{CN}$ and $\mathrm{OH}$. The list of non-detections is considerably longer and includes such interesting species as $\mathrm{H}_{2} \mathrm{O}, \mathrm{C}_{3}, \mathrm{MgH}, \ldots$ (see Table 1 of van Dishoeck and Black 1988a for a summary). The first need for atomic and molecular data in diffuse cloud studies lies in the availability 
of high-resolution visible and ultraviolet laboratory spectra to identify the species. In addition, accurate oscillator strengths for the electronic transitions are required to convert the measured equivalent widths into column densities. Specific needs are discussed in the paper by J.H. Black in this volume.

There are two other classes of interstellar clouds that are chemically very similar to the classical diffuse clouds, but which are studied by different techniques. Translucent clouds with $A_{V} \approx 2-5 \mathrm{mag}$ are "thicker" versions of the classical diffuse clouds (Crutcher 1985; van Dishoeck and Black 1988b). The molecular clouds detected at high galactic latitudes have $A_{V}=1-2 \mathrm{mag}$, similar to the diffuse clouds (Magnani, Blitz and Mundy 1985). Both types of clouds have large enough molecular column densities to allow millimeter observations of $\mathrm{CO}$ and species such as $\mathrm{CS}, \mathrm{HCN}, \mathrm{H}_{2} \mathrm{CO}, \mathrm{NH}_{3}$ and $\mathrm{H}_{2} \mathrm{CO}$. On the other hand, these clouds are still thin enough to permit absorption line observations, provided that there is a bright star located fortuitously behind the cloud. The interpretation of the millimeter data requires accurate rotational excitation cross sections, both by $\mathrm{H}_{2}$ and by electrons. Only very limited information is currently available on electron-molecule excitation rates at low temperatures.

\section{CHEMICAL MODELS}

The basic outline of the chemistry responsible for the formation of the simplest molecules was established already more than 15 years ago. Detailed models for comparison with observations were first made by Black and Dalgarno (1977), whereas more recent studies include those of van Dishoeck and Black (1986) and Viala, Roueff and Abgrall (1988). With the well-known exception of $\mathrm{CH}^{+}$, the models initially were able to reproduce the observed abundances quite well using reasonable parameters for unknown reaction rate coefficients. However, now that more accurate reaction rates are becoming available through detailed laboratory or theoretical work, it becomes increasingly difficult to develop models that are in harmony with the complete set of observational data for a cloud.

The modeling of a specific interstellar cloud consists of two steps:

\subsection{Physical conditions}

Since the reaction rates depend on temperature, density and the intensity of the radiation field, the physical conditions need to be constrained as closely as possible before the chemistry can be studied. In diffuse clouds, the observed excitations of various species are used as diagnostics, and accurate data on the atomic and molecular processes that enter the excitation calculations are needed.

The primary diagnostic on which most diffuse cloud models are based is $\mathrm{H}_{2}$. The low rotational levels $J<3$ of this molecule are populated primarily by collisional processes and are thus sensitive to temperature; the higher levels $J>5$ are thought to be populated mostly by an ultraviolet pumping process, so that their populations are sensitive to the strength of the interstellar radiation field. The relative abundance of atomic to molecular hydrogen depends on the density in the cloud. Although the rates for collisional excitation of $\mathrm{H}_{2}$ by $\mathrm{H}_{2}$ are fairly well determined (Schaefer 1985; Danby, Flower and Monteiro 1987), the $\mathrm{H}_{2}-\mathrm{H}$ cross sections are still uncertain by an order of magnitude (Green and Truhlar 1979; Allison and Dalgarno 1967). Better information on this system is badly needed.

Other important diagnostics in diffuse clouds include:

- $\mathrm{C}_{2}$ : the rotational levels of this homonuclear molecule are also populated by a combination of collisional and optical pumping processes, followed by infrared cascade (van 
Dishoeck and Black 1982). The population distribution over the lower levels is again sensitive to temperature, whereas that over the higher levels depends on the ratio of the density and the strength of the radiation field in the red. Better information on the rotational excitation cross sections of $\mathrm{C}_{2}$ by $\mathrm{H}_{2}$ is needed, although theoretical calculations for this system have recently been reported by Chambaud et al. (1988). Note also that in diffuse clouds, the abundance of ortho- $\mathrm{H}_{2}(J=1)$ is comparable to that of para- $\mathrm{H}_{2}(J=0)$, so that excitation rates by both species are required. Another uncertain molecular parameter in the $\mathrm{C}_{2}$ excitation calculation is the $\mathrm{C}_{2} \mathrm{a}^{3} \Pi_{u}-\mathrm{X}^{1} \Sigma_{g}^{+}$ intercombination transition moment.

- CO: its rotational levels are populated primarily by collisional processes so that the excitation is sensitive to both density and temperature. Collisional rates of $\mathrm{CO}$ with para $-\mathrm{H}_{2}(J=0)$ are now well determined (Schinke et al. 1985; Flower and Launay $1985)$, but rates with ortho- $\mathrm{H}_{2}(J=1)$ are still uncertain.

- Atomic species: the excitation of the fine structure levels of the $\mathrm{C}^{+}, \mathrm{C}$ and $\mathrm{O}$ atoms occurs mostly by collisions with atomic and molecular hydrogen, and with electrons. Although theoretical calculations of rates for collisions with $\mathrm{H}$ have been available for some time, the rates for collisions with $\mathrm{H}_{2} \quad(J=0,1)$ are based mostly on educated guesses. Monteiro and Flower (1987) have recently pointed out that $J=0 \rightarrow 1$ excitation of $\mathrm{C}$ and $\mathrm{O}$ by $\mathrm{H}_{2}(J=0)$ is in first order forbidden. Accurate calculations for the $\mathrm{C}^{+}$ $e$ cross sections exist (Hayes and Nussbaumer 1984), but not for the C- $e$ or O- $e$ collisions. Experimental information on all of these systems would be most welcome.

In diffuse clouds, the various diagnostics give physical conditions that are consistent within factors of a few, although it is often not possible to construct a unique model. The temperature in the models typically varies from $>100 \mathrm{~K}$ at the edge to $30 \mathrm{~K}$ in the center, and the density from $100-200 \mathrm{~cm}^{-3}$ at the edge to $500-1000 \mathrm{~cm}^{-3}$ in the center. The largest uncertainty currently lies in the strength of the interstellar radiation field, which is constrained only by the $\mathrm{H}_{2}$ high- $J$ population under the assumption that ultraviolet pumping is the dominant excitation mechanism. Typically, the radiation field is found to be enhanced over the average field by factors of 3-5. As discussed in the paper by Hartquist in this volume, the levels may also be populated by collisions in shock-heated gas, in which case the inferred radiation fields are only upper limits.

\subsection{Chemical network}

The second step in the modeling consists of solving the steady-state chemical network at each depth. Integration of the concentrations over depth then yields the column densities. Overviews of the chemistry in diffuse clouds can be found e.g. in van Dishoeck and Black (1988a), van Dishoeck (1988a) and Black and Dalgarno (1977). Detailed information on the rate coefficients for the reactions that enter the networks is needed at this stage of the modeling procedure. It should be realized, however, that not all reactions are equally important, so that not all rate coefficients need to be known with equally high precision. It is the task of the astrochemists to identify the crucial reactions for which accurate rate coefficients are required. In the following, some of these crucial reactions with poorlydetermined rate coefficients will be mentioned.

\subsubsection{Carbon chemistry}

The formation of the carbon-bearing molecules is thought to be initiated by the radiative association reaction

$$
\mathrm{C}^{+}+\mathrm{H}_{2} \rightarrow \mathrm{CH}_{2}^{+}+\mathrm{h} \nu \text {. }
$$




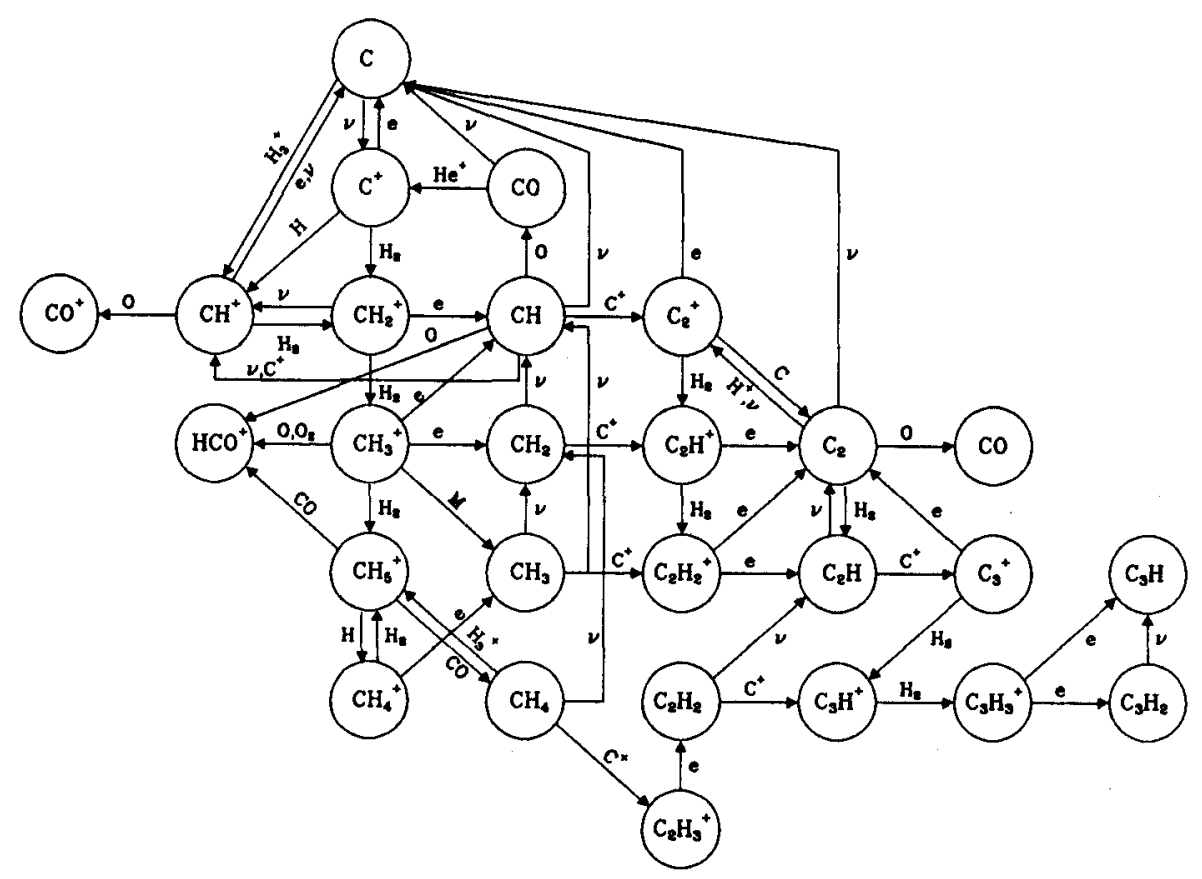

Figure 1. The most important chemical reactions involving carbon-bearing molecules. $M$ stands for metal.

Although this reaction was postulated more than 15 years ago (Black and Dalgarno 1973), it has still not been measured in the laboratory, nor is there any theoretical calculation accurate to better than an order of magnitude. The approach of the astrochemists has therefore been to use the observed $\mathrm{CH}$ abundance to infer a value for this rate. The models for the $\varsigma$ Per and $\varsigma$ Oph diffuse clouds require $k_{r a} \approx 5 \times 10^{-16} \mathrm{~cm}^{3} \mathrm{~s}^{-1}$, a value which is consistent with the theoretial estimate of $k_{r a}=10^{-16}-10^{-15} \mathrm{~cm}^{3} \mathrm{~s}^{-1}$ (Herbst 1982), but which is close to the experimental upper limit $k_{r a}<1.5 \times 10^{-15} \mathrm{~cm}^{3} \mathrm{~s}^{-1}$ at $13 \mathrm{~K}$ (Luine and Dunn 1985). Note, however, that this experimental limit pertains to the reaction of $\mathrm{C}^{+}$with normal hydrogen having an ortho:para ratio of $3: 1$, whereas the interstellar models require the rate for an ortho:para ratio of about 1:2. Radiative association with ortho- $\mathrm{H}_{2}$ is expected to be slower than that with para- $\mathrm{H}_{2}$ on theoretical grounds. An experimental verification of the astronomically determined rate is urgently needed. Even an upper limit lowered by a factor of four compared with that of Luine and Dunn (1985) would provide significant tests of the carbon chemistry

Further down in the carbon-chemistry network (see Figure 1), the dissociative recombination reaction

$$
\begin{aligned}
\mathrm{CH}_{3}^{+}+\mathrm{e} & \rightarrow \mathrm{CH}_{2}+\mathrm{H} \\
& \rightarrow \mathrm{CH}+\mathrm{H}_{2} \text { or } \mathrm{H}+\mathrm{H}
\end{aligned}
$$

occurs. In this case, the reaction rate coefficient itself is not very important (as long as the reaction is rapid), but the branching ratio to form $\mathrm{CH}_{2}$ and $\mathrm{CH}$ significantly affects the 
chemistry (see Black and van Dishoeck 1988 for an overview). If the branching ratio favors $\mathrm{CH}_{2}$, the abundance of $\mathrm{CH}_{2}$ is increased significantly in the models, and consequently also the abundances of subsequent molecules in the chain, such as $\mathrm{C}_{2}$ and $\mathrm{C}_{2} \mathrm{H}$. Similarly, the branching ratios of the dissociative recombination of $\mathrm{C}_{3} \mathrm{H}_{3}^{+}$to form cyclic or linear $\mathrm{C}_{3} \mathrm{H}_{2}$ and $\mathrm{C}_{3} \mathrm{H}$ are important. Virtually no theoretical or experimental information on these branching ratios is currently available from experiment or theory.

The principal destruction mechanism of all neutral molecules in diffuse clouds is photodissociation. Many of the photo rates adopted in the early diffuse cloud models were based on educated guesses. Over the last ten years, more accurate rates have been determined for a number of species through detailed calculations or laboratory experiments. In most cases, the new rates are significantly larger than previous estimates, making it more difficult to reproduce the measured abundances. The photodissociation and photoionization rates for interstellar molecules have recently been summarized by van Dishoeck (1988b), and specific needs have been pointed out. In the carbon chemistry, the photo rates of the $\mathrm{CH}_{2}, \mathrm{C}_{2} \mathrm{H}$ and $\mathrm{C}_{3} \mathrm{H}_{2}$ molecules are particularly uncertain. In addition, the branching ratios to dissociation and ionization following absorption of photons with energies greater than the ionization potential of the molecule are not well known.

\subsubsection{Oxygen chemistry}

The formation of oxygen-bearing molecules is driven either by the near-resonant chargetransfer reaction

$$
\mathbf{O}+\mathrm{H}^{+} \rightarrow \mathrm{O}^{+}+\mathrm{H}
$$

or

$$
\mathrm{O}+\mathrm{H}_{3}^{+} \rightarrow \mathrm{OH}^{+}+\mathrm{H}_{2} \text {. }
$$

Reaction (4) proceeds rapidly at low temperature, but the rate coefficient for reaction (3) is more uncertain, and only theoretical estimates are available (Chambaud et al. 1980). Whether reaction (3) or (4) dominates the formation route depends on the abundance of $\mathrm{H}_{3}^{+}$in diffuse clouds. This abundance, in turn, depends on the rate at which $\mathrm{H}_{3}^{+}$is removed by dissociative recombination:

$$
\mathrm{H}_{3}^{+}+\mathbf{e} \rightarrow \mathrm{H}_{2}+\mathrm{H} \text { or } 3 \mathrm{H} \text {. }
$$

The rate coefficient of reaction (5) for $\mathrm{H}_{3}^{+}$in its lowest vibrational level $v=0$ is currently very controversial. Although there was significant evidence from both theoretical and experimental sides that this process may be very slow under interstellar conditions (Michels and Hobbs 1984; Adams and Smith 1987), more recent experiments suggest that it remains rapid at low temperatures (Amano 1988; Hus et al. 1988). A discussion of the possible origin for these discrepancies has been given by Smith and Adams (1988). If $k_{5}<1^{-9}$ $\mathrm{cm}^{3} \mathrm{~s}^{-1}$, reaction (4) dominates the oxygen chemistry.

Other important molecular data needed in the oxygen chemistry include the branching ratios for dissociative recombination, in particular for $\mathrm{H}_{3} \mathrm{O}^{+}$to form $\mathrm{H}_{2} \mathrm{O}$; the rate at which $\mathrm{C}^{+}$reacts with the polar molecule $\mathrm{OH}$ to form $\mathrm{CO}$ at low temperatures; and the photodissociation rates of the neutral species. Of particular importance is the rate at which $\mathrm{CO}$ photodissociates. Recent laboratory work on the vacuum ultraviolet absorption spectrum of ${ }^{12} \mathrm{CO}$ by Letzelter et al. (1987) and Yoshino et al. (1988) has clarified significantly the nature of its photodissociation processes, but crucial information on the isotopic species ${ }^{13} \mathrm{CO}, \mathrm{C}^{18} \mathrm{O}$ and ${ }^{13} \mathrm{C}^{18} \mathrm{O}$ is still lacking. A summary of the spectroscopic information needed for $\mathrm{CO}$ has been given by van Dishoeck and Black (1988c). 


\subsubsection{Nitrogen chemistry}

The formation routes for nitrogen-bearing molecules in diffuse clouds are particularly uncertain. Previous models assumed that the principal initiating route is the exothermic reaction

$$
\mathrm{N}+\mathrm{H}_{3}^{+} \rightarrow \mathrm{NH}_{2}^{+}+\mathrm{H} \text {. }
$$

Theoretical calculations (Herbst et al. 1987), however, indicate that there are large barriers along the reaction path so that the rate is likely to be slow at low temperatures. An experimental upper limit would be very useful. The other initiating reaction

$$
\mathrm{N}^{+}+\mathrm{H}_{2} \rightarrow \mathrm{NH}^{+}+\mathrm{H}
$$

is also likely to be slow under interstellar conditions (Rowe 1988). Alternative gas-phase reactions through which nitrogen-containing molecules can be formed include neutralneutral reactions of atomic nitrogen with small oxygen- and carbon-containing molecules such as $\mathrm{OH}, \mathrm{CH}$ and $\mathrm{C}_{2}$ to form $\mathrm{CN}$ and $\mathrm{NO}$, and ion-molecule reactions of atomic nitrogen with small hydrocarbons to form e.g. $\mathrm{H}_{2} \mathrm{CN}^{+}$, which can then recombine to form $\mathrm{CN}$ and HCN. Experimental information on the rate coefficients of the neutral-neutral reactions at low temperatures is lacking.

Neutral molecules such as CN are rapidly destroyed by photodissociation and by reactions with atomic oxygen. Experimental determinations of the reaction rate coefficients for the latter processes would be useful.

\section{SUMMARY}

Atomic and molecular data are needed in the modeling of diffuse clouds at three different stages:

- accurate transition wavelengths and oscillator strengths are required to identify the species and to convert the measured equivalent widths into column densities.

- accurate collisional excitation rates of various diagnostic species are needed to constrain the physical conditions.

- accurate rate coefficients for crucial reactions are needed to test the chemistry.

Although significant progress has been made in various areas, there are still a number of processes, such as dissociative recombination and radiative association, for which reliable experimental or theoretical results are only just beginning to appear. The models are therefore not yet conclusive as to whether the adopted chemical network is complete and whether steady-state gas-phase models can explain the abundances of most molecules in diffuse clouds. The current diffuse cloud models can reproduce the abundances of a number of the observed molecules such as $\mathrm{CH}, \mathrm{C}_{2}, \mathrm{OH}$ and $\mathrm{HD}$ with reasonable, although ad hoc values for rates of crucial reactions such as (1) and (5). On the other hand, the models tend to produce too little $\mathrm{CO}$ and $\mathrm{CN}$ compared with observations by factors of a few. These discrepancies most likely result from an overestimate of the strength of the interstellar radiation field incident on the model clouds. However, until more accurate data on the crucial atomic and molecular processes are available, we cannot be confident that we fully understand the chemistry of the simplest diatomic molecules in diffuse interstellar clouds. 


\section{REFERENCES}

Adams, N.G. and Smith, D. 1987, in IAU Symposium 120, Astrochemistry, eds. M.S. Vardya and S.P. Tarafdar (Reidel, Dordrecht), p. 1.

Allison, A.C. and Dalgarno, A. 1967, Proc. Phys. Soc. London, 90, 609.

Amano, T. 1988, Ap. J. (Letters), 329, L121.

Black, J.H. 1987, in IAU Symposium 120, Astrochemistry, eds. M.S. Vardya and S.P. Tarafdar (Reidel, Dordrecht), p. 217.

Black, J.H. and Dalgarno, A. 1973, Astrophys. Letters, 15, 79.

Black, J.H. and Dalgarno, A. 1977, Ap. J. Suppl., 34, 405.

Black, J.H. and van Dishoeck, E.F. 1988, to appear in Dissociative Recombination: theory, experiments and applications, eds. J.B.A. Mitchell and S.L. Guberman (World Scientific Publishing, Singapore).

Chambaud, G., Launay, J.M., Levy, B., Millie, P., Roueff, E., and Tran Minh, F. 1980, J. Phys. B, 13, 4205.

Chambaud, G., Lavendy, H., Levy, B., Robbe, J.M., and Roueff, E. 1988, Chem. Phys., submitted.

Crutcher, R.M. 1985, Ap. J., 288, 604.

Crutcher, R.M. and Watson, W.D. 1985, in Molecular Astrophysics, eds. G.H.F. Diercksen et al., NATO ASI Series 157 (Reidel, Dordrecht), p. 255.

Dalgarno, A. 1988, Astro. Lett. and Communications, 26, 153.

Danby, G., Flower, D.R., and Monteiro, T.S. 1987, M. N. R. A. S., 226, 739.

Flower, D.R. and Launay, J.M. 1985, M. N. R. A. S., 214, 271.

Green, S. and Truhlar, D.G. 1979, Ap. J. (Letters), 231, L101.

Hayes, M.A. and Nussbaumer, H. 1984, Astr. Ap., 134, 193.

Herbst, E. 1982, Ap. J., 252, 810.

Herbst, E., DeFrees, D.J., and McLean, A.D. 1987, Ap. J., 321, 898.

Hus, H., Yousseif, F., Sin, A., and Mitchell, J.B.A. 1988, Phys. Rev. A, in press.

Letzelter, C., Eidelsberg, M., Rostas, F., Breton, J., and Thieblemont, B. 1987, Chem. Phys., 114, 273.

Luine, J.A. and Dunn, G.H. 1985, Ap. J. (Letters), 299, L67.

Magnani, L., Blitz, L., and Mundy, L. 1985, Ap. J., 295, 402.

Michels, H.H. and Hobbs, R.H. 1984, Ap. J. (Letters), 286, L27.

Monteiro, T.S. and Flower, D.R. 1987, M. N. R. A. S., 228, 101.

Rowe, B.R. 1988, in Rate Coefficients in Astrochemistry, eds. T.J. Millar and D. Williams (Kluwer, Dordrecht), p. 135.

Schaefer, J. 1985, in Molecular Astrophysics, eds. G.H.F. Diercksen et al., NATO ASI Series 157 (Reidel, Dordrecht), p. 497.

Schinke, R., Engel, V., Buck, U., Meyer, H., and Diercksen, G.H.F. 1985, Ap. J., 299, 939.

Smith, D. and Adams, N.G. 1988, to appear in Dissociative Recombination: theory, experiments and applications, eds. J.B.A. Mitchell and S.L. Guberman (World Scientific Publishing, Singapore).

van Dishoeck, E.F. 1988a, to appear in Molecular Astrophysics - A volume honoring Alexander Dalgarno, ed. T.W. Hartquist (Cambridge University).

van Dishoeck, E.F. 1988b, in Rate Coefficients in Astrochemistry, eds. T.J. Millar and D. Williams (Kluwer, Dordrecht), p. 49.

van Dishoeck, E.F. and Black, J.H. 1982, Ap. J., 258, 533.

van Dishoeck, E.F. and Black, J.H. 1986, Ap. J. Suppl., 62, 109.

van Dishoeck, E.F. and Black, J.H. 1988a, in Rate Coefficients in Astrochemistry, eds. 
T.J. Millar and D. Williams (Kluwer, Dordrecht), p. 209. van Dishoeck, E.F. and Black, J.H. 1988b, Ap. J., submitted. van Dishoeck, E.F. and Black, J.H. 1988c, Ap. J., Nov. 15 issue. Viala, Y.P., Roueff, E., and Abgrall, H. 1988, Astr. Ap., 190, 215.

Yoshino, K., Stark, G., Smith, P.L., Parkinson, W.H., and Ito, K. 1988, in preparation. 